\title{
DOS TEMPOS DE COLÔNIA À DEMOCRACIA ATUAL: UMA ANÁLISE SOBRE ARRANJOS INSTITUCIONAIS E DESIGUALDADE SOCIAL NA AMÉRICA LATINA
}

\author{
Luiza Amelotti ${ }^{1}$ \\ Evertton Lira ${ }^{2}$
}

\begin{abstract}
Resumo
Qual o efeito dos arranjos institucionais sobre a desigualdade social? Regimes democráticos podem ser definidos como uma série de regras e procedimentos que objetivam transferir poder político e acomodar conflitos. Uma vez que o sistema esteja mais consolidado e permita maior acesso ao poder, é esperado que isso se traduza em menores níveis de desigualdade. O objetivo deste artigo é testar o efeito do desenho institucional dos países ao longo da história sobre a desigualdade social. Nossas hipóteses são, de maneira geral, que (a) países com colonização menos extrativista e (b) com regimes democráticos mais sólidos e plurais têm menor desigualdade em função de suas instituições. Para testar essas hipóteses, empregamos modelos de regressão em painel, utilizando como unidade de análise os países da América Latina no período 1980-2019. Os principais resultados apontam que a democracia tem efeitos negativos sobre a pobreza e a desigualdade de renda. Da mesma forma, países que se constituíram sob grandes desigualdades sociais continuaram reproduzindo suas instituições e, consequentemente, a desigualdade.
\end{abstract}

Palavras-chave: América Latina. Instituições. Desigualdade. Pobreza.

\begin{abstract}
What is the effect of institutional design on social inequality? Democratic regimes can be defined as a series of rules and procedures that aim to transfer political power and settle conflicts. Once the system is more consolidated and allows greater access to power, it is expected this will translate into lower levels of inequality. The purpose of this article is to test the effect of countries' institutional design throughout history on social inequality. Our hypotheses are, in general, that (a) countries with less extractive colonization and (b) with more solid and plural democratic regimes show less inequality as an outcome of their institutions. To test these hypotheses, we used panel regression models, taking Latin American countries as the unit of analysis in the period 1980-2019. The main results point out that democracy has negative effects on poverty and income inequality. Likewise, countries that were constituted under great social inequalities continued to reproduce their institutions and, consequently, inequality.
\end{abstract}

Key-words: Latin America. Institutions. Inequality. Poverty.

\section{Resume}

¿Cuál es el efecto de los diseños institucionales sobre la desigualdad social? Los regímenes democráticos se pueden definir como una serie de reglas y procedimientos que tienen como objetivo transferir el poder político y resolver los conflictos. Una vez que el sistema es más consolidado y permite un mayor acceso al poder, se espera que esto se traduzca en menores niveles de desigualdad. El propósito de este artículo es probar el efecto del diseño institucional de los países a lo largo de la historia sobre la desigualdad social. Nuestras hipótesis son, en general, que (a) países con menos colonización extractiva y (b) con regímenes democráticos más sólidos y plurales presentan menos desigualdad según sus instituciones. Para probar estas hipótesis, utilizamos modelos de regresión de panel, tomando países de América Latina como unidad de análisis en el período 1980-2019. Los principales resultados señalan que la democracia tiene efectos

\footnotetext{
${ }^{1}$ Doutoranda, mestre e graduada em Ciência Política pela Universidade Federal de Pernambuco (UFPE).
}

${ }^{2}$ Doutorando, mestre e graduado em Ciência Política pela Universidade Federal de Pernambuco (UFPE). 
negativos sobre la pobreza y la desigualdad de renta. Asimismo, países que se constituyeron bajo grandes desigualdades sociales continuaron reproduciendo sus instituciones y, en consecuencia, la desigualdad.

Palabras-clave: América Latina. Instituiciones. Desigualdad. Pobreza.

\section{INTRODUÇÃO}

A literatura sobre democracia tem passado por diversas transformações conceituais. Uma das definições mais clássicas é a democracia enquanto um método, um procedimento (SCHUMPETER, 1942). Essa definição mínima tem consequências normativas importantes: se a democracia é apenas um método de seleção de governos, o desenho institucional desse regime não tem importância para a produção de resultados sociais e políticos.

Para englobar a complexidade de o que significa ter um regime democrático, podemos definir a democracia enquanto "a body of rules and procedures that regulates the transfer of political power and the free expression of disagreement at all levels of public life" (TAVARES, WACZIARG, 2001, p. 1342). Pensar o sistema enquanto um conjunto de regras que permite a transferência de poder político e a acomodação de conflitos é útil para compreender os efeitos dos diversos arranjos institucionais sobre resultados políticos.

Um dos elementos que caracterizam o sistema democrático é o seu potencial de inclusão. $\mathrm{O}$ acesso da população mais pobre ao poder por meio do voto livre e universal (DIAMOND, MORLINO, 2004) levaria a uma transformação das elites políticas e, consequentemente, a influência no processo político (TAVARES, WACZIARG, 2001).

Seguindo essa linha de raciocínio, nosso argumento é que desenhos institucionais que permitam maior acesso ao poder produzem resultados sociais mais inclusivos. O mecanismo adjacente a esse argumento é que instituições com menores barreiras à entrada (com menores custos) elevariam a participação de atores de fora das tradicionais elites política e econômica. Isso produziria ambientes institucionais com maior participação da sociedade, tornando o atendimento de suas demandas mais eficientes (CASTRO, 1991). Em comparação com as oligarquias, os regimes democráticos produzem maior crescimento econômico no longo prazo, visto que trazem consigo garantias de acesso a direitos iguais e consequente redução das barreiras de entrada, ao contrário do que se apresenta nos sistemas oligárquicos (ACEMOGLU, 2003).

Para responder à pergunta "qual o efeito dos arranjos institucionais sobre a desigualdade social?”, nossa análise foca nos países latino-americanos no período 1980-2019. O recorte temporal de cada país inicia-se apenas no ano em que se tornaram um regime democrático. A escolha dessa região tem algumas razões: em primeiro lugar, um relatório publicado pela Organização das Nações 
Unidas aponta que a América Latina é a região mais desigual do mundo ${ }^{3}$. Em segundo lugar, a literatura aponta que a região tem processos políticos particulares, que se distinguem das premissas de homogeneidade global (MAINWARING, PÉREZ-LIÑAN, 2003; 2007). Essas informações indicam a relevância de buscar compreender os determinantes institucionais de indicadores tão preocupantes.

\section{POBREZA E DESIGUALDADE NA AMÉRICA LATINA}

Pobreza e desigualdade de renda são problemas de grande escala em numerosos lugares do mundo, mas em especial na América Latina. A região apresenta os maiores níveis de desigualdade entre todas as regiões em desenvolvimento do planeta, mesmo após a vertiginosa queda ocorrida a partir dos anos 2000 (IDB, 2020; RAVALLION, 2016). Da mesma forma, a incidência da pobreza é historicamente alta quando comparada a outras regiões de renda per capita similar (IDB, 2020).

As definições de pobreza podem variar no espaço e no tempo, bem como em relação à sua métrica (ALI KHAN, 2013). Mensurações de pobreza a partir da definição de "insuficiência de renda" levam em consideração valores mínimos necessários para satisfazer necessidades básicas de alimentação, vestuário e abrigo para garantir a sobrevivência do indivíduo (TODARO, 2002). Desigualdade, por sua vez, diz respeito à disparidade nos padrões de vida existente entre um conjunto de indivíduos (RAVALLION, 2016). Apesar de conceitualmente distintas, pobreza e desigualdade caminham juntas. As duas, junto ao crescimento econômico, fazem parte de um triângulo de interdependência (BOURGUIGNON, 2004). Ambas se afetam mutuamente.

A história da desigualdade na América Latina aponta que nem sempre a região foi tão desigual (WILLIAMSON, 2015). No século XV, a região viveu os menores índices de desigualdades já registrados (idem). Com a chegada dos colonizadores europeus, a redistribuição de terras para as elites teve um forte efeito negativo sobre o desenvolvimento econômico e a distribuição de renda (ENGERMAN, SOKOLOFF, 2005).

As potências ibéricas instalaram nos países latino-americanos instituições com poucos direitos à propriedade privada, com grande foco extrativista (ACEMOGLU, JOHNSON, ROBINSON, 2001). A persistência dessas instituições ao longo do tempo fez com que a distribuição de renda fosse menos responsiva a ciclos econômicos favoráveis (FRANKEMA, 2005).

\footnotetext{
3 https://www.latinamerica.undp.org/content/rblac/en/home/presscenter/pressreleases/2019/unfair-and-unequal-new-undp-report-sheds-light-on-discontent-in.html
} 
Diferenças significativas são encontradas, também, entre as colonizações portuguesa e espanhola. Evidências empíricas para o Brasil mostram que, após a divisão pelo Tratado de Tordesilhas, o lado controlado por Portugal recebeu um número muito maior de negros escravizados que o lado espanhol. Resultados de um modelo de regressão descontínua apontam que os municípios muito próximos da linha estipulada pelo tratado têm indicadores de desigualdade consideravelmente diferentes: do lado português, maior escravidão e desigualdade. Do lado espanhol, menor escravidão e desigualdade (FUJIWARA, LAUDARES, VALENCIA, 2017).

Tais níveis de desigualdade são, também, efeitos de desenhos institucionais e, ao mesmo tempo, exercem influência sobre as instituições. Sobre o primeiro ponto, a literatura aponta que sistemas descentralizados são mais propensos à redução da pobreza e desigualdades (VON BRAUN, GROTE, 2000; CRAWFORD, 2008). Por sua vez, regimes politicamente fechados retardam o crescimento econômico e a redução da pobreza (ROBINSON, ACEMOGLU, 2012), assim como instituições economicamente disfuncionais que servem à elite perpetuam a pobreza e contribuem para aumentar a desigualdade (RAVALLION, 2016).

A existência de níveis tão alarmantes de desigualdades sociais tem consequências, também, sobre a democracia. Recursos econômicos podem ser convertidos em recursos políticos (DAHL, 1996) e ao acesso a diversos setores burocráticos (SANTOS, 2001). Da mesma forma, a nãoparticipação efetiva dos mais pobres tem efeito negativo sobre a legitimidade da democracia (BENNETTT, 1986; DE LUCA, 1995). Em resumo, a efetividade dos direitos civis é perturbada pela desigualdade econômica (KERSTENETZKY, 2002).

\section{ORIGEM COLONIAL E FORMAÇÃO DAS DESIGUALDADES NA AMÉRICA LATINA}

A formação dos Estados modernos repousa sobre as tensões entre as elites que defendiam a expansão do poder dos funcionários reais e as que pressionavam o governo por representação política (CARVALHO, 1996). De tais embates, se desenvolveram instituições centrais à legitimidade e à natureza de poder desses Estados, entre as quais estão a burocracia, o monopólio do uso da força, o ordenamento jurídico e o poder sobre o território (CARVALHO, 1996). Entre as principais inovações desse período estavam o desenvolvimento do corpo burocrático, bem como dos Parlamentos nacionais (CARVALHO 1996).

Se, por um lado, o estabelecimento do Estado moderno europeu contou com um longo período de transformações e adaptações, por outro, nas ex-colônias da América Latina, o processo foi muito mais célere, dependente de forças exógenas e instável (CARVALHO, 1996). Na dinâmica 
capitalista, os Estados latino-americanos continuavam imersos numa relação de dependência dos países centrais.

Esse movimento dificultou o controle das sociedades dependentes sobre o desenvolvimento e acentuou o desequilíbrio social herdado do período colonial (SAMPAIO JR., 1999). No panorama da América Latina, podemos observar instituições que permaneceram de pé muitos anos após a fase do colonialismo, como, por exemplo, o trabalho escravo que, no Brasil, durou até 1886; no México e na Guatemala, foi reintroduzido e se manteve, respectivamente, até 1910 e 1945 (ACEMOGLU, JOHNSON, ROBINSON, 2001).

\footnotetext{
"Although we commonly described the independent polities as 'new states', in reality they were successors to the colonial regime, inheriting its structures, its quotidian routines and practices, and its more hidden normative theories of governance" (YOUNG, 1994 apud ACEMOGLU, JOHNSON, ROBINSON, 2001, p. 1376).
}

Não somente as instituições, mas também as elites são heranças do período colonial. No caso brasileiro, essa classe encontrou melhores condições para a construção do novo Estado, dando continuidade a um cenário já definido e garantindo um "aparato estatal mais organizado, mais coeso, e talvez mesmo mais poderoso" (CARVALHO 1996, p. 40). Esse fato, associado à escravidão, levou a um maior controle das elites sobre a sociedade e, em consequência, reduziu as chances ocorrer mobilidade social ascendente (CARVALHO, 1996).

Dessa maneira, as formas de exploração desenvolvidas nas colônias, as instituições que dela emergiram, e a sua formação social foram determinantes na construção dos Estados independentes. Desde o ano de 1776, há registros na literatura de investigações sobre os legados coloniais.

Após diversas tipologias e classificações, os neoinstitucionalistas Engerman e Sokoloff (1997) apontaram três modelos coloniais: (i) colônias agrícolas com alta capacidade de exportação (Brasil, Cuba e países caribenhos); (ii) colônias em que nativos e europeus tiveram que conviver e se adaptar (México e Peru); e (iii) colônias com poucos nativos e baixa produção agrícola (Argentina, Uruguai e Costa Rica). Para cada uma dessas classificações, as instituições locais foram formuladas e adaptadas para atender a realidade em que se instalavam.

No primeiro modelo indicado, a economia era de escala, foi muito difundido o sistema de plantation e a utilização do trabalho escravo africano. "Esta situação inicial gerou instituições “ruins”, que perduraram até mesmo depois da abolição da escravidão" (MONASTERIO, EHRL, 1990, p. 21). Assim, as elites foram atraídas pela oportunidade de exploração da terra, mas não buscavam se estabelecer nesses territórios. Portanto, trouxeram consigo muitos escravos africanos e as instituições que se formavam estavam amparadas por uma clivagem extrema: de um lado a elite rica exploradora, do outro a mão de obra escrava africana e nativa. 
No segundo tipo, os nativos sobreviveram aos europeus e compuseram grande parte da população. Se estabeleceram instituições extrativistas e altamente excludentes; com a prática de encomenda, a elite dominante recolhia tributos e empobrecia ainda mais a população local (ENGERMAN, SOKOLOFF, 1997). Nesses territórios, a elite tinha algum interesse de estabelecer e desenvolver e, apesar da alta desigualdade social - principalmente entre imigrantes e nativos houve espaço para a formação de diferentes classes sociais.

Esse modelo converge com o das Colônias de Conquista (ROSCHER, 1856), as quais giravam em torno da exploração dos nativos. O caso chileno é indicado por Roscher (1856) como mais um exemplo dessa categoria (MONASTERIO, EHRL, 1990).

Finalmente, as colônias do terceiro tipo, não atraiam interesse e investimentos da metrópole. Nesses territórios, se estabeleceram mais trabalhadores livres em busca de terras, levando a uma menor desigualdade social. Tais fatores aproximam esta categoria aos modelos de Colônias de Povoamento (MONASTERIO, EHRL, 1990). Aqui, também se encaixa o Paraguai, um território mais isolado e que não contava com muitas riquezas, permanecendo subdesenvolvido e pouco povoado (HANRATTY, 2005).

Quadro 1 - Tipos de colônias

\begin{tabular}{||c|c|c||}
\hline Tipo de Colônia & Sociedade & Países \\
\hline $\begin{array}{c}\text { Poucos nativos e baixa } \\
\text { produtividade }\end{array}$ & $\begin{array}{c}\text { Baixo desenvolvimento, } \\
\text { porém menos desigualdade } \\
\text { social }\end{array}$ & $\begin{array}{c}\text { Argentina, Uruguai, Costa } \\
\text { Rica, Paraguai }\end{array}$ \\
\hline Exploração de nativos & $\begin{array}{c}\text { Altamente desigual } \\
\text { (Imigrantes x Nativos) }\end{array}$ & $\begin{array}{c}\text { México, Peru, Chile, } \\
\text { Venezuela, Bolívia, Equador }\end{array}$ \\
\hline Economia de escala & $\begin{array}{c}\text { Extremamente desigual } \\
\text { (Elite x Escravos) }\end{array}$ & $\begin{array}{c}\text { Brasil, Cuba, Haiti, Rep. } \\
\text { Dominicana, Colômbia }\end{array}$ \\
\hline
\end{tabular}

Fonte: Adaptado de Engerman e Sokoloff (2005).

\section{DEMOCRACIA E REDUÇÃO DAS DESIGUALDADES}

Governos democráticos são mais propensos a batalhas por redistribuição e redução das desigualdades. Em democracias, as elites políticas têm incentivos eleitorais para melhorar a qualidade de vida dos menos favorecidos (BALCÁZAR, 2016). Da mesma forma, têm mecanismos 
institucionais que permitem mudanças no poder político, incluindo as classes médias e outros segmentos menos favorecidos que demandam por mais recursos e distribuição nas arenas decisórias (LIPSET, 1959; MELTZER, RICHARD, 1981).

Existem alguns mecanismos que compõem essa relação. O primeiro diz respeito a taxações e transferências: quanto maior a distância da renda mediana entre os grupos representados, maior é a demanda por redistribuição. Segundo, quanto mais políticos competem por apoio popular, maior é a quantidade de serviços públicos prestados. Como a democracia induz à competição, ela melhoraria os serviços ofertados. Terceiro, a democracia favorece a livre associação entre trabalhadores, encorajando a sindicalização (TIMMONS, 2010).

$\mathrm{Na}$ América Latina, houve uma emergência de democracias na segunda metade do século XX, favorecendo o declínio observado na desigualdade dos países da região (HUBER, STEPHENS, 2012). Desde a crise econômica que se estendeu pela década de 1930, elites conservadoras alcançaram o poder e a região passou por períodos que intercalam governos autoritários e democráticos (BETHELL, 1997). Havia, também, pouco gasto social de qualquer tipo no século XIX e início do século XX, principalmente porque a voz na política era muito restrita (LINDERT, 2004). No entanto, durante o fim do século XX, as democracias começaram a ficar mais consolidadas e mais partidos de esquerda chegaram ao poder, adotando políticas progressivas e redistributivas (LUSTIG, 2007; HUBER, STEPHENS, 2012).

O alto nível de desigualdade no período pós-colonial exacerbou problemas de ação coletiva e financiamento da educação. As elites proviam educação apenas para si mesmas e resistiam às taxações que pudessem subsidiar a educação (ENGERMAN, SOKOLOFF, 2002). A onda democrática do fim do século XX levou à expansão do ensino público de base, aumentando os níveis educacionais dos cidadãos (LÓPEZ-CALVA, LUSTIG, 2010; HUBER, STEPHENS, 2012). Esta expansão aumentou a produtividade da economia (SAINT-PAUL, VERDIER, 1993; BOURGUIGNON, VERDIER 2000).

O efeito da democracia sobre a redução das desigualdades, no entanto, é percebido no longo prazo. Governos democráticos mais antigos permitem maior distribuição de recursos (HUBER, STEPHENS, 2012), são politicamente mais estáveis e com experiência acumulada. É preciso que a democracia esteja estabelecida há um tempo considerável para que a desigualdade de fato caia (MULLER, 1988).

Democracias, portanto, levam ao empoderamento dos cidadãos (CHRISTOFOROU, 2010). Por sua vez, a participação popular impulsionada pelo empoderamento leva a direcionamento nas políticas públicas para estes setores. Por exemplo, maior participação pode afetar diretamente a 
saúde pública, (GERRING ET AL., 2020). Da mesma maneira, democracias são positivamente correlacionadas com aumento das receitas, gastos sociais em educação, saúde, seguridade social, (PERSSON, TABELLINI, 2003; HUBER, STEPHENS, 2012) e crescimento econômico (ACEMOGLU, ROBINSON, 2012; ACEMOGLU ET AL., 2015). Todos esses fatores formam a ligação entre a democracia e a redução das desigualdades possível. Na próxima seção, apresentamos as hipóteses deste trabalho.

\section{HIPÓTESES}

A partir das discussões anteriormente apresentadas, este trabalho tem quatro hipóteses sobre a relação entre desenho institucional e desigualdades sociais:

H1: O tipo de colonização caracterizado pela "economia de escala" gera sociedades mais desiguais quando comparado a outros tipos de colônias.

Com base na literatura apresentada na seção 3, países estabelecidos nessas bases tiveram colonização marcada pelo extrativismo e instituições com pouca ou nenhuma proteção aos direitos de propriedade, bem como alto grau de escravidão. Há uma grande disparidade entre as elites políticas e econômicas e o restante da população, reduzindo fortemente o potencial redistributivo da democracia.

H2: Quanto maior o nível de democracia do país, menores são as desigualdades sociais.

Níveis mais elevados de democracia estão associados a uma maior absorção de preferências do eleitorado, maior acesso à educação e adoção de políticas redistributivas, reduzindo as desigualdades.

H3: Quanto maior a experiência democrática em anos, menores são as desigualdades sociais.

Democracias mais antigas são mais consolidadas, com maior experiência e aperfeiçoamento de suas instituições. Por sua vez, são mais capazes de lidar com problemas sociais de longo prazo.

\section{DESENHO DE PESQUISA}

Para testar as hipóteses acima, utilizamos como unidade de análise todos os países latinoamericanos no período 1980-2019. A inclusão de cada país, no entanto, só é feita a partir do momento em que se tornou uma democracia. Por exemplo: o Brasil voltou a ter um regime democrático apenas em 1985, portanto, suas informações são contabilizadas a partir daí. 


\subsection{VARIÁVEIS DEPENDENTES}

Para mensurar o conceito de desigualdade social, optamos por utilizar indicadores de pobreza e GINI. Ambos são importantes para compreender melhor o quadro da desigualdade social numa determinada região. Para a variável "pobreza”, usamos dados do Banco Mundial sobre o percentual de população que vive com menos de US $\$ 1,90$ por dia (medida em Paridade do Poder de Compra). Essa é uma mensuração adequada para nossos propósitos, uma vez que torna os países comparáveis e está de acordo com as linhas nacionais absolutas de pobreza (RAVALLION, CHEN, SANGRAULA, 2009).

A segunda variável dependente, índice de GINI, é um indicador largamente utilizado e reconhecido que aponta a diferença entre os rendimentos dos mais ricos e dos mais pobres. $\mathrm{Ou}$ seja, é uma medida de concentração de renda. Varia de 0 a 1, onde o valor máximo representaria o maior nível de desigualdade possível. Os dados também foram coletados do Banco Mundial.

\subsection{VARIÁVEIS INDEPENDENTES}

Utilizamos quatro variáveis independentes (VI) para mensurar a evolução ao longo da história do desenho institucional do país. São elas:

- Origem colonial: variável categórica ordinal mensurada segundo o quadro 1: 1- Poucos nativos e baixa produtividade, 2- Exploração de nativos e 3- Economias de escala (ver quadro 2. Fonte: adaptado de Engerman e Sokoloff, 1997). A ordenação desse indicador parte de um tipo de colônia estabelecida sob menos desigualdade e vai até àquela que detinha extrema desigualdade.

- Nível de democracia: mensurada numa escala de 1 a 10, em que 1 é o nível mais frágil e 10 é uma democracia forte (fonte: Quality of Government Institute Standard Data, 2020 )

- Tempo cumulativo de experiência democrática: mensurada a partir do total de anos que o país esteve sob um regime democrático (fonte: Quality of Government Institute Standard Data, 2020)

\subsection{VARIÁVEIS DE CONTROLE}

- Nível de participação da sociedade na política: mensurada numa escala de 0 a 1 , é calculada a partir de indicadores de seleção de candidatos, consultas à sociedade civil, ambiente de participação e participação das mulheres (fonte: $V-D e m^{5}$, 2020)

\footnotetext{
${ }^{4}$ Teorell, Jan, Aksel Sundström, Sören Holmberg, Bo Rothstein, Natalia Alvarado Pachon \& Cem Mert Dalli. 2020. The Quality of Government Standard Dataset, version Jan20. University of Gothenburg: The Quality of Government Institute, http://www.qog.pol.gu.se doi:10.18157/qogstdjan20.

${ }^{5}$ Coppedge, Michael, John Gerring, Carl Henrik Knutsen, Staffan I. Lindberg, Jan Teorell, Nazifa Alizada, David Altman, Michael Bernhard, Agnes Cornell, M. Steven Fish, Lisa Gastaldi, Haakon Gjerløw, Adam Glynn, Allen Hicken,
} 
- Número Efetivo de Partidos (NEP): calculado a partir da divisão de 1 sobre o somatório do quadrado das proporções de cadeiras dos partidos no legislativo (fonte: $V$-Dem, 2020).

- Taxa de mortalidade infantil: calculada a partir dos óbitos de 0 a 5 anos por mil nascimentos (fonte: Gapminder, 2020).

- Crescimento econômico: porcentagem da variação anual do PIB em relação ao ano anterior (fonte: Banco Mundial, 2020).

- Crescimento populacional: porcentagem da variação no crescimento da população em relação ao ano anterior (fonte: Banco Mundial, 2020).

- Anos de escolaridade: média dos anos estudados da população maior de 15 anos de idade (fonte: $V$-Dem, 2020).

\subsection{TÉCNICAS ESTATÍSTICAS}

Para testar a relação entre as variáveis acima, utilizamos técnicas de estatística descritiva, gráfico de dispersão, correlação de Pearson e regressão linear em painel com efeitos aleatórios. ${ }^{6} \mathrm{~A}$ escolha de efeitos aleatórios foi feita em função da maior capacidade desse modelo em estimar os coeficientes a partir de variáveis com baixa variação (CLARK, LINZER, 2012), a exemplo da VI = origem colonial. Um modelo de efeito fixo iria excluir automaticamente essa variável, já que ela é fixa no espaço e no tempo. Um problema adicional é a natureza e distribuição dos dados ao longo do tempo, uma vez que as duas dependentes têm tendência decrescente. Consequências disso são problemas de não-estacionaridade, correlação serial, heteroscedasticidade, heterogeneidades espacial e temporal. Contra esses problemas, adicionamos coeficientes robustificados e também um efeito two-ways aos modelos que foram estimados utilizando o software R Studio.

\section{RESULTADOS}

Como é possível notar na imagem 1, a América Latina tem uma trajetória de redução dos níveis de pobreza e desigualdade ao longo da série histórica. Nas décadas de 1980 e 1990 há uma grande volatilidade dos dados, mostrando períodos de grandes elevações e quedas nos índices. Os

Garry Hindle, Nina Ilchenko, Joshua Krusell, Anna Luhrmann, Seraphine F. Maerz, Kyle L. Marquardt, Kelly McMann, Valeriya Mechkova, Juraj Medzihorsky, Pamela Paxton, Daniel Pemstein, Josefine Pernes, Johannes von Römer, Brigitte Seim, Rachel Sigman, Svend-Erik Skaaning, Jeffrey Staton, Aksel Sundström, Ei-tan Tzelgov, Yi-ting Wang, Tore Wig, Steven Wilson and Daniel Ziblatt. 2020. "V-Dem [Country-Year/Country-Date] Dataset v10" Varieties of Democracy Project. https://doi.org/10.23696/vdemds20.

${ }^{6}$ No teste de multicolinearidade (Variance Inflation Factor), todas as variáveis apresentaram coeficiente menor que 3.70, o que indica ausência do problema no modelo (James et al., 2013). Da mesma maneira, apesar de o teste de ShapiroWilk indicar a não normalidade dos resíduos, isso só seria um problema relevante caso a amostra fosse $\mathrm{N}<100$ (Gujarati e Porter, 2011). 
anos 1980 foram marcados por uma forte crise econômica na região, impulsionada pela forte dívida externa dos países, produzindo uma "década perdida" (CAMPELLO, ZUCCO JR., 2016). Na década de 1990, começou alguma estabilidade econômica, o que iria contribuir para alguma redução, mas os países voltaram a ter altos níveis de pobreza e desigualdade ao fim da década. A partir dos anos 2000, com a ascensão de governos de esquerda na região (HUBER e STEPHENS, 2012) e o boom dos preços de commodities (CAMPELLO, ZUCCO, 2016) houve uma queda acentuada nos níveis de pobreza e desigualdade.

Imagem 1 - Média das variáveis dependentes
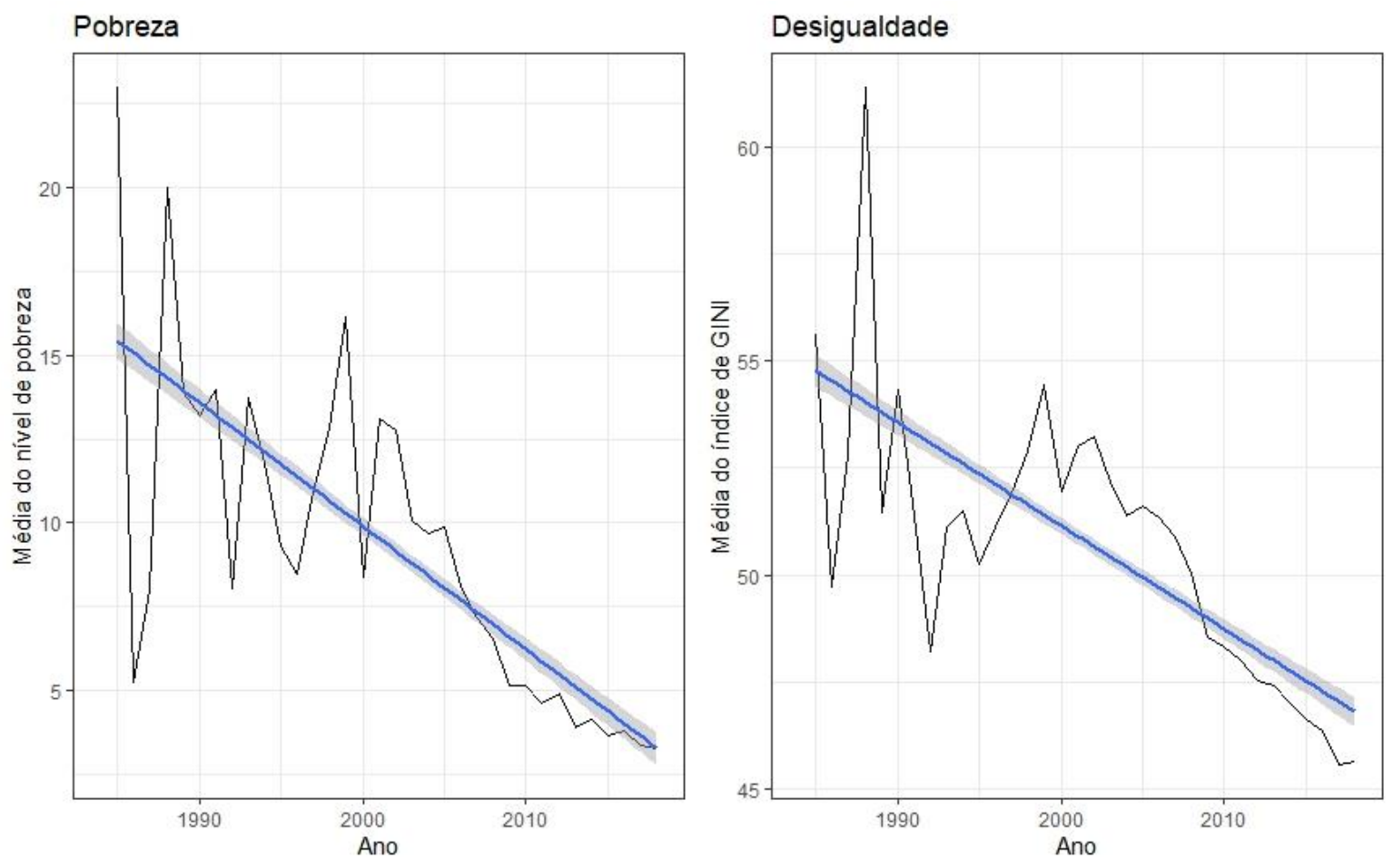

Fonte: Elaboração própria.

Como já mencionamos, pobreza e desigualdade são problemas que caminham juntos. A construção histórica dos países latino-americanos, como também em outras regiões do mundo, engloba o estabelecimento de elites políticas e econômicas com grande concentração de terras e privilégios. Ao mesmo tempo, o estabelecimento de instituições voltadas aos interesses dessas elites dificultou a ascensão das camadas mais pobres. O resultado desses processos, somados a escravidão no continente, levaram a um elevado número de população vivendo sob pobreza extrema e grande concentração de renda nas elites. A imagem 2 é um scatterplot da distribuição das duas variáveis e 
evidencia uma forte relação positiva entre elas. O coeficiente de correlação de Pearson é 0,69 , com um $\mathrm{p}$ valor estatisticamente significante $(\mathrm{p}<0,05)$.

Imagem 2 - Scatterplot das variáveis dependentes

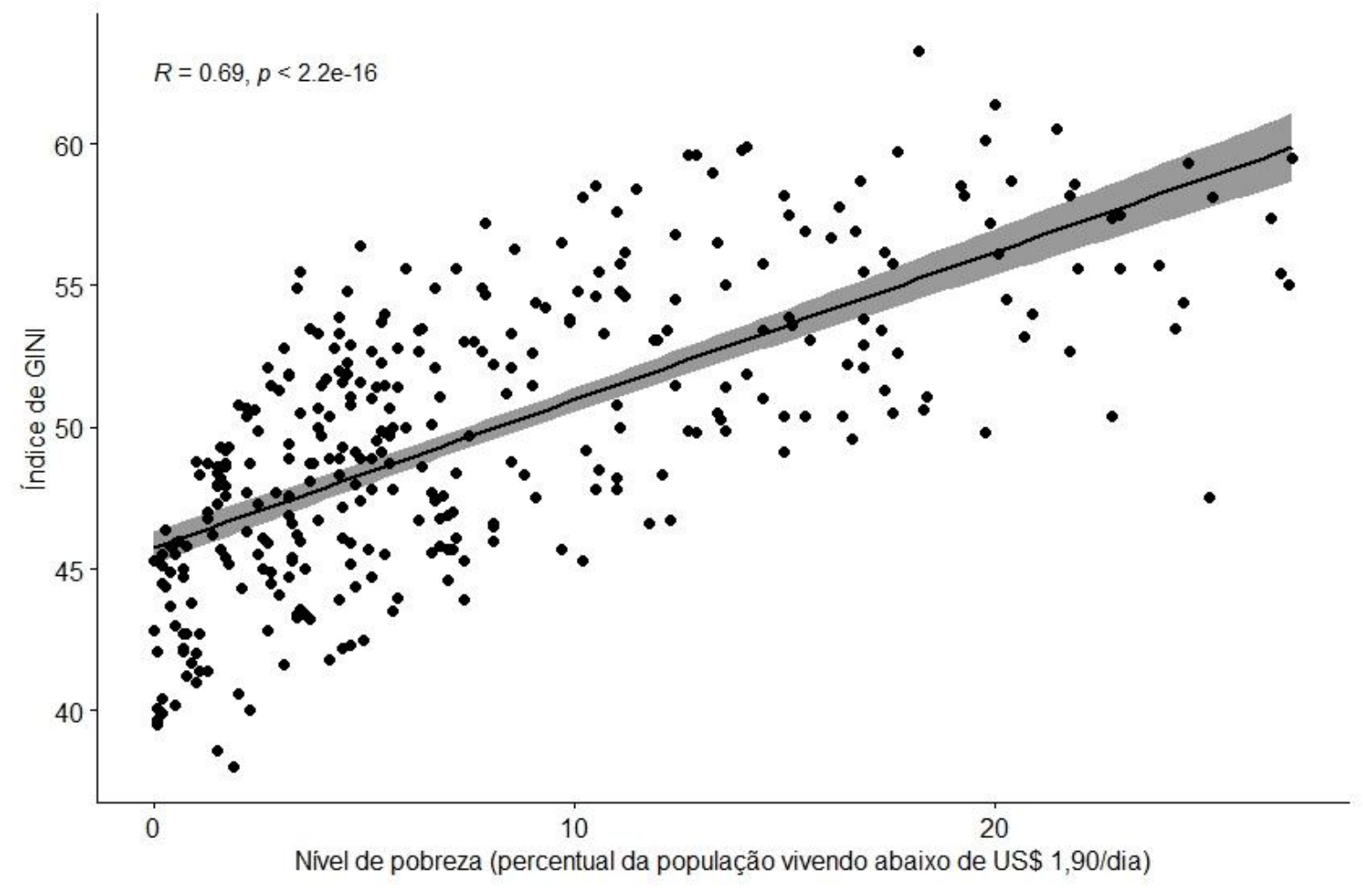

Fonte: Elaboração própria.

$\mathrm{Na}$ tabela 1 abaixo, apresentamos os coeficientes dos modelos de regressão em painel com efeitos aleatórios analisados para as variáveis pobreza e indice de GINI.

Tabela 1 - Resultados dos modelos de regressão

\begin{tabular}{c|c|c|c|c}
\hline \multirow{2}{*}{} & \multicolumn{4}{c}{ Variáveis dependentes } \\
\cline { 2 - 5 } & Pobreza (1) & $\begin{array}{c}\text { Erros robustos - } \\
\text { pobreza (2) }\end{array}$ & GINI (3) & $\begin{array}{c}\text { Erros robustos - } \\
\text { GINI (4) }\end{array}$ \\
\hline Origem & $1.203^{* *}$ & $\begin{array}{c}1.203^{* *} \\
(0.608)\end{array}$ & $\begin{array}{c}2.092^{* * *} \\
(0.307)\end{array}$ & $\begin{array}{c}2.092^{* * *} \\
(0.521)\end{array}$ \\
\hline $\begin{array}{c}\text { Delonial } \\
\text { (nível) }\end{array}$ & $\begin{array}{c}-0.360^{* * *} \\
(0.035)\end{array}$ & $\begin{array}{c}-0.360^{* * *} \\
(0.130)\end{array}$ & $\begin{array}{c}-0.072^{* *} \\
(0.035)\end{array}$ & $\begin{array}{c}-0.072 \\
(0.145)\end{array}$ \\
\hline $\begin{array}{c}\text { Democracia } \\
\text { (anos) }\end{array}$ & $\begin{array}{c}-0.080^{* * *} \\
(0.017)\end{array}$ & $\begin{array}{c}-0.080^{* * *} \\
(0.030)\end{array}$ & $\begin{array}{c}-0.058^{* * *} \\
(0.012)\end{array}$ & $\begin{array}{c}-0.058^{* *} \\
(0.024)\end{array}$ \\
\hline
\end{tabular}




\begin{tabular}{|c|c|c|c|c|}
\hline Participação & $\begin{array}{c}-10.162^{* * *} \\
(1.149)\end{array}$ & $\begin{array}{c}-10.162^{* * *} \\
(3.369)\end{array}$ & $\begin{array}{l}-1.711 \\
(1.079)\end{array}$ & $\begin{array}{l}-1.711 \\
(3.166)\end{array}$ \\
\hline NEP & $\begin{array}{c}0.413 * * * \\
(0.047)\end{array}$ & $\begin{array}{c}0.413^{* * *} \\
(0.185)\end{array}$ & $\begin{array}{c}0.245^{* * *} \\
(0.046)\end{array}$ & $\begin{array}{c}0.245 \\
(0.170)\end{array}$ \\
\hline$\Delta$ População & $\begin{array}{c}1.875^{* * *} \\
(0.321)\end{array}$ & $\begin{array}{c}1.875^{* * *} \\
(0.691)\end{array}$ & $\begin{array}{c}-1.336^{* * *} \\
(0.281)\end{array}$ & $\begin{array}{c}-1.336^{*} \\
(0.730)\end{array}$ \\
\hline$\Delta \mathrm{PIB}$ & $\begin{array}{c}-0.147 * * * \\
(0.020)\end{array}$ & $\begin{array}{l}-0.147 \\
(0.105)\end{array}$ & $\begin{array}{c}0.002 \\
(0.020)\end{array}$ & $\begin{array}{c}0.002 \\
(0.072)\end{array}$ \\
\hline $\begin{array}{l}\text { Mortalidade } \\
\text { infantil }\end{array}$ & $\begin{array}{c}0.095^{* * *} \\
(0.010)\end{array}$ & $\begin{array}{c}0.095^{* * *} \\
(0.036)\end{array}$ & $\begin{array}{c}0.115^{* * * *} \\
(0.009)\end{array}$ & $\begin{array}{c}0.115^{* * *} \\
(0.033)\end{array}$ \\
\hline $\begin{array}{l}\text { Anos de } \\
\text { escolaridade }\end{array}$ & $\begin{array}{c}-2.285^{* * *} \\
(0.276)\end{array}$ & $\begin{array}{c}-2.285^{* * *} \\
(0.402)\end{array}$ & $\begin{array}{l}-0.233 \\
(0.189)\end{array}$ & $\begin{array}{l}-0.233 \\
(0.369)\end{array}$ \\
\hline Constante & $\begin{array}{c}30.350 * * * \\
(2.385)\end{array}$ & $\begin{array}{c}30.350^{* * *} \\
(4.678)\end{array}$ & $\begin{array}{c}50.147 * * * \\
(1.803)\end{array}$ & $\begin{array}{c}50.147 * * * \\
(4.329)\end{array}$ \\
\hline
\end{tabular}

\begin{tabular}{c|c|c|c|c}
\hline Observações & 328 & \multirow{5}{*}{} & 326 \\
\cline { 1 - 2 } R2 & 0.510 & & 0.334 \\
\cline { 1 - 2 } R2 ajustado & 0.496 & & 0.315 \\
F Statistic & $1,751.714^{* * *}$ & & $492.478^{* * *}$ & \\
\hline
\end{tabular}

Nota: ${ }^{*} \mathrm{p}<0.1,{ }^{* *} \mathrm{p}<0.05,{ }^{* * *} \mathrm{p}<0.01$

Fonte: Elaboração Própria.

Os modelos demonstram que a origem colonial apresenta efeitos significativos, tanto para o índice de GINI, quanto para a pobreza e, como esperado, países classificados como "economia de escala" - categoria 3: colônia marcada pela desigualdade extrema - apresentam maiores índices das duas variáveis dependentes. Isso indica que os Estados que se consolidaram sobre sistemas fortemente exclusivos, voltados para a exploração da terra através do trabalho escravo, tendem a reproduzir, em certa medida, os altos níveis de desigualdade social, como consequência da formação de suas instituições e elites dominantes. Já aqueles países que se constituíram sobre sociedades formadas, em grande parte, de homens livres em busca de melhores condições de vida (categoria 1: menor desigualdade), tendem a desenvolver um desenho institucional que vise à maior absorção das demandas sociais. Esses resultados condizem com a literatura (ENGERMAN, SOKOLOFF, 2002; 2005). A imagem 3 apresenta boxplots das variáveis dependentes categorizadas 
pelo tipo de origem colonial. Assim como os modelos indicam, o efeito é maior para o nível de desigualdade, sobretudo na categoria 3.

Imagem 3 - Boxplot das variáveis dependentes classificados por tipo de origem colonial
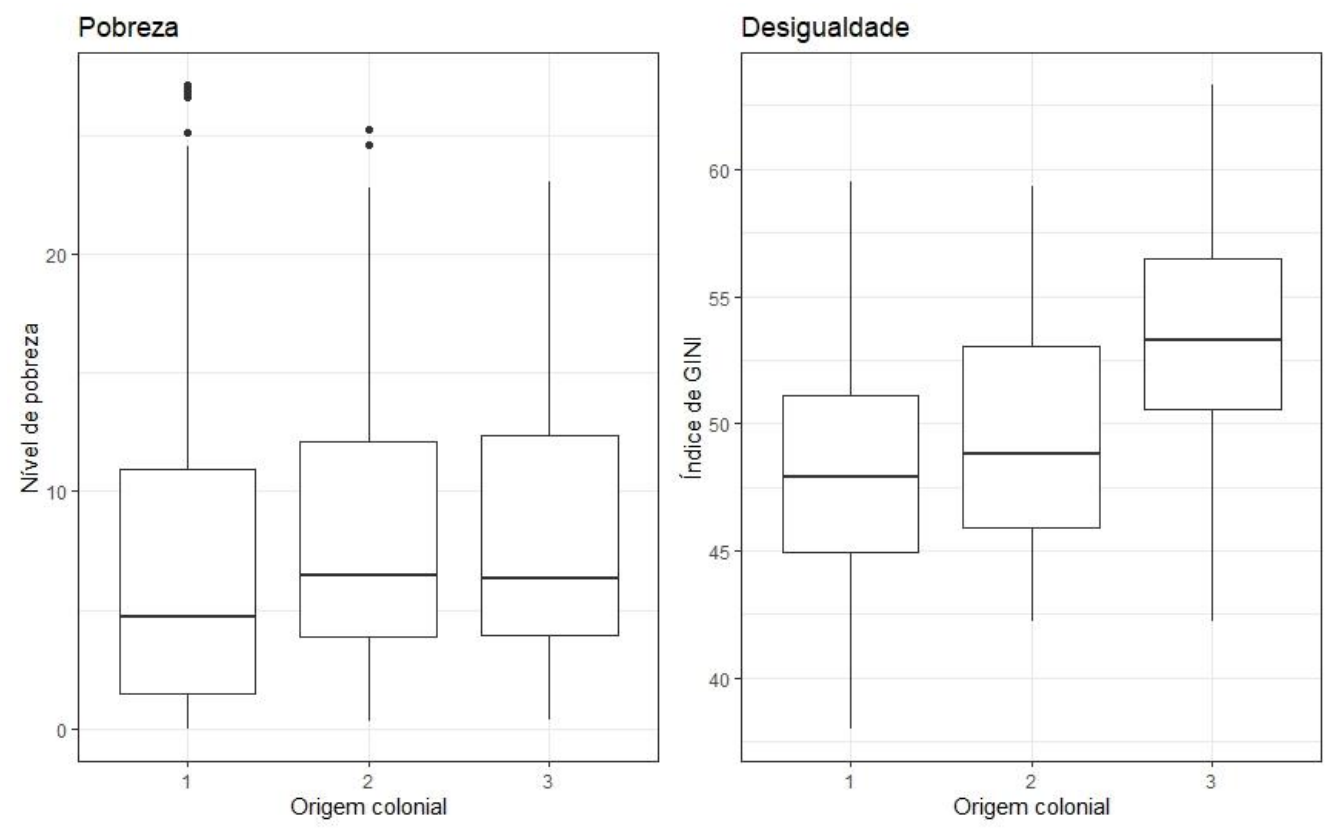

Fonte: Elaboração própria.

Em segundo lugar, o nível de democracia tem efeitos negativos significativos sobre os índices de pobreza, ou seja, quanto mais democrático o Estado, menor o percentual da população vivendo com renda abaixo de US\$1,90 por dia. Esse resultado corrobora nossas expectativas, as quais indicam que as demandas do eleitorado, o acesso à educação e a adoção de políticas redistributivas tendem fatores mais observados em regimes mais democráticos. O efeito sobre a desigualdade também é negativo e significante, porém perde significância quando aplicado o erro padrão robusto. As duas variáveis condizem com a literatura, uma vez que é esperado que haja mais redistribuição à medida que a democracia se desenvolva.

Já o impacto da consolidação da democracia, analisada pela soma dos anos desse regime por país, se mostrou negativo e significativo para ambas variáveis de interesse, confirmando as hipóteses de que quanto mais antigas, mais experientes são, de modo que podem aperfeiçoar suas instituições para lidarem com problemas sociais, reduzindo a pobreza e garantindo mais oportunidades à população, o que leva a uma redução também da desigualdade.

As variáveis de controle, por sua vez, indicaram que: (i) a participação afeta negativamente a pobreza, o que pode ser explicado pelo argumento de que uma sociedade mais participativa garante 
maior acesso às políticas redistributivas; (ii) o número efetivo de partidos no legislativo afeta positivamente o nível de pobreza e de desigualdade. Esse efeito, porém, tem sentido inverso ao esperado. Tal evidência lança luz sobre a questão de que a quantidade de partidos talvez não seja condição suficiente à representação de todos os grupos sociais e que a representação de fato se torna restrita às classes dominantes. Esse argumento se relaciona, ainda, com a origem colonial dos países, ou seja, se o Estado se consolidou sobre instituições focadas em atender às elites, é possível que a configuração institucional democrática traga consigo características herdadas nesse período.

O indicador de crescimento (iii) $\Delta$ População teve um efeito significativo sobre a pobreza e GINI, sendo positivo para a primeira para a segunda. Há uma relação positiva entre taxas de fertilidade mais altas e pobreza nos países. O crescimento das famílias afeta negativamente a renda disponível para os membros que constituem aquele núcleo, uma vez que recursos já escassos precisam ser divididos para mais pessoas (DUFLO, BANERJEE, 2011), contribuindo com a reprodução intergeracional da pobreza. Esse processo leva à armadilha da pobreza (RAVALLION, 2016), criando um cenário onde a família não dispõe de recursos para financiar melhores serviços de saúde e educação, encarando também a privação do acesso à moradia, alimentação e vestuário. O segundo indicador de crescimento, (iv) $\triangle P I B$, apresentou efeito significativo apenas sobre a pobreza, indicando que quanto maior a riqueza do país, maior sua capacidade produtiva e de geração de empregos.

Como proxy para o desenvolvimento humano, foi utilizado a (v) taxa de mortalidade infantil, que apresentou efeitos positivos sobre as duas dependentes. Esse indicador representa a ponta final de uma série de políticas públicas voltadas para a promoção de saúde e desenvolvimento, que são essenciais para a superação da pobreza e das desigualdades. Como mencionado anteriormente, a perpetuação destes problemas está ligada a armadilhas que podem ser combatidas mediante políticas públicas mais eficazes.

Finalmente, (vi) anos de escolaridade teve impacto negativo e significante sobre o nível de pobreza, corroborando as expectativas de que mais educação impulsiona mais acesso a empregos com maiores salários e à qualificação profissional. Outra forma que a educação leva a uma redução da pobreza está relacionado ao conhecimento que as pessoas desenvolvem acerca de seus direitos, da estruturação de políticas e do funcionamento das instituições, de forma que suas demandas por acesso, redistribuição, saúde e saneamento, por exemplo, ganham mais força. 


\section{CONCLUSÕES}

Para responder a questão "qual o efeito dos arranjos institucionais sobre a desigualdade social?", analisamos os impactos das variáveis nível de democracia, consolidação democrática e origem colonial sobre os níveis de pobreza e desigualdade dos países latino-americanos.

A escolha da região se deu pelo fato dela apresentar os maiores níveis de desigualdade entre todas as regiões em desenvolvimento do planeta, bem como pela alta incidência da pobreza quando comparada a outras regiões de renda per capita similar (IDB, 2020; RAVALLION, 2016). Além disso, esse fenômeno não é recente na América Latina: a chegada dos colonizadores europeus, a redistribuição de terras para as elites e a formação de instituições tiveram um forte efeito negativo sobre o desenvolvimento econômico e a distribuição de renda (ENGERMAN, SOKOLOFF, 2005; FRANKEMA, 2005).

Os males herdados do período colonial continuaram se mostrando como barreiras ao avanço social, dado que as elites proviam educação apenas para si mesmas e resistiam às taxações que pudessem subsidiar a educação (ENGERMAN, SOKOLOFF, 2002). Contudo, com a emergência das democracias, na segunda metade do século XX, observou-se um declínio no nível de desigualdade dos países da região (HUBER, STEPHENS, 2012).

Diante disso, nossos argumentos são de que países que foram colônias intensamente extrativistas e que suas instituições não garantiam devida proteção aos direitos de propriedade e perpetuavam um alto grau de escravidão, irão, em alguma medida, reproduzir as desigualdades reduzindo fortemente o potencial redistributivo da democracia. No entanto, quanto mais consolidado o regime democrático, mais experiência e capacidade de aperfeiçoamento dessas instituições, bem como, níveis mais elevados de democracia estarão associados a uma maior absorção de preferências do eleitorado, maior acesso à educação e adoção de políticas redistributivas. Juntos, esses dois aspectos tendem a ter bons efeitos para combater tais problemas sociais.

Através do modelo de regressão em painel com efeitos aleatórios, as hipóteses obtiveram respaldo estatístico: o nível e a consolidação da democracia tiveram efeitos significativos e negativos sobre a pobreza e a desigualdade; e a origem colonial teve impacto significativo e positivo sobre as duas variáveis. Desse modo, percebe-se que o desenvolvimento dos países latino-americanos, desde os primeiros momentos de sua construção como Estado, traz consigo características apreendidas ao longo de sua história e que, em certa medida, continuam reproduzindo efeitos sobre a sua configuração social. O advento da democracia foi de grande importância para o combate e a 
redução das mazelas sociais, mas ainda há muito que se avançar na América Latina para superação da pobreza e desigualdade.

Mesmo com as evidências apontadas através dos modelos, pobreza e desigualdade são temas difíceis de explicar. Existem diversas forças políticas, sociais, institucionais, econômicas e territoriais que podem contribuir para a perpetuação desses problemas. Por definição, já configura uma limitação deste trabalho. Além disso, o efeito das instituições coloniais sobre a desigualdade no período democrático recente tende a apresentar efeitos dissipativos no tempo, o que poderia até mesmo subestimar nossas estimativas. Portanto, se faz necessário mais pesquisas sobre o tema, especialmente no sentido de tentar conectar as forças mencionadas anteriormente e apontar evidências mais robustas.

\section{REFERÊNCIAS}

ACEMOGLU, D. 2003. Why not a political Coase theorem? Social conflict, commitment, and politics. Journal of comparative economics, vol. 31, n 4, p. 620-652.

ACEMOGLU, D., JOHNSON, S., ROBINSON, J. A. 2001. The colonial origins of comparative development: An empirical investigation. American economic review, vol. 91, nº 5, p. 1369-1401.

ACEMOGlU, D., NAIDU, S., RESTREPO, P., ROBINSON, J. A. 2015. Democracy, redistribution, and inequality. In: Handbook of income distribution (vol. 2, p. 1885-1966). Elsevier.

ALI KHAN, S. 2013. Decentralization and poverty reduction: A theoretical framework for exploring the linkages. International Review of Public Administration, vol. 18, $\mathrm{n}^{\circ}$ 2, p. 145-172. https://doi.org/10.1080/12294659.2013.10805256

BALCÁZAR, C. F. 2016. Long-run effects of democracy on income inequality in Latin America. The Journal of Economic Inequality. https://doi.org/10.1007/s10888-016-9329-3

BENNETT, S. E. 1986. Apathy in America - 1960/1984 - Causes and Consequences of Citizen Political Indifference. New York, Transnational Publishers, Inc.

BETHELL, L. 1997. Historia de América Latina: Politica y Sociedad desde 1930. In: Historia de America Latina. Barcelona: Critica (Grijalbo Mondadori).

BOURGUIGNON, F. 2004. The poverty-growth-inequality triangle (No. 125). working paper.

BOURGUIGNON, F., VERDIER, T. 2000. Oligarchy, democracy, inequality and growth. Journal of development Economics, vol. 62, n² 2, p. 285-313.

CAMPELLO, D., ZUCCO JR. C. 2016. Presidential Success and the World Economy. The Journal of Politics, vol. $78, \mathrm{n}^{\circ} 2$. 
CARVALHO, J. M. de. 1996. A construção da ordem e Teatro de sombras. Rio de Janeiro: Editorial da UFRJ/Relume Dumará.

CASTRO, M. H. G. D. 1991. Descentralização e política social no Brasil: as perspectivas dos anos 90. Espaço e Debates, vol. 100, n 32.

CLARK, T. S., \& LINZER, D. A. 2015. Should I use fixed or random effects. Political Science Research and Methods, vol. 3, n 2, p. 399-408.

CRAWFORD, G. 2008. Decentralization and the limits to poverty reduction: Findings from Ghana. Oxford Development Studies, vol. 36, n² 2, p. 235-258.

DAHL, R. A. 1996. Equality versus inequality. PS: Political Science and Politics, vol. 29, n 4, p. 639648.

DE LUCA, T. 1995. The Two Faces of Political Apathy. Philadelphia: Temple University Press.

DIAMOND, L., MORLINO, L. 2004. The quality of democracy: An overview. Journal of democracy, vol. $15, \mathrm{n}^{\circ} 4$, p. $20-31$.

DUFLO, E., \& BANERJEE, A. 2011. Poor economics: A radical rethinking of the way to fight global poverty. PublicAffairs.

ENGERMAN, S. L., SOKOLOFF, K. 1997. Factor endowments, institutions, and differential paths of growth among new world economies: a view from economic historians of the United States. In: HABER, S. How Latin America Fell Behind. Stanford CA: Stanford University Press.

ENGERMAN, S. L., SOKOLOFF, K. L. 2002. Factor endowments, inequality, and paths of development among new world economics. $\mathrm{N}^{\circ}$ 9259, NBER Working Papers, National Bureau of Economic Research, Inc.

ENGERMAN, S. L., SOKOLOFF, K. L. 2005. Colonialism, inequality, and long-run paths of development. N 11057, NBER Working Papers, National Bureau of Economic Research, Inc.

FRANKEMA, E. H. 2005. The colonial origins of inequality: Exploring the causes and consequences of land distribution (No. 119). Discussion papers//Ibero America Institute for Economic Research.

FUJIWARA, T., LAUDARES, H., CAICEDO, F. V. 2017. Tordesillas, slavery and the origins of Brazilian inequality. Sl: sn.

GERRING, J., KNUTSEN, C. H., MAGUIRE, M., SKAANING, S. E., TEORELL, J., COPPEDGE, M. 2020. Democracy and human development: issues of conceptualization and measurement. Democratization, 1-25.

GUJARATI, D. N., PORTER, D. C. 2011. Econometria básica. Amgh Editora.

HANRATTY, D. M. 2005. A Country Profile. In: Washington, DC: Federal Research Division for the Library of Congress. Retrieved October. p. 2008. 
HUBER, E., STEPHENS, J. D. 2012. Democracy and the left: Social policy and inequality in Latin America. University of Chicago Press.

INTER-AMERICAN DEVELOPMENT BANK. 2020. The Inequality Crisis: Latin America and the Caribbean at the crossroads.

JAMES, G., WITTTEN, D., HASTIE, T., TIBSHIRANI, R. 2013. An introduction to statistical learning. New York: springer.

KERSTENETZKY, C. L. 2002. Por que se importar com a desigualdade. Dados, vol. 45, n4, p. 649-675.

LINDERT, P. H. 2004. Growing public: Volume 1, the story: Social spending and economic growth since the eighteenth century (Vol. 1). Cambridge University Press.

LIPSET, S. M. 1959. Some social requisites of democracy: Economic development and political legitimacy. The American political science review, vol. 53, n 1, p. 69-105.

LÓPEZ-CALVA, L. F., LUSTIG, N. 2010. Explaining the decline in inequality in Latin America: Technological change, educational upgrading and democracy. In: LÓPEZ-CALVA, L. F., LUSTIG, N. Declining Inequality in Latin America: a decade of progress. Brookings Institution Press, 124.

LUSTIG, N. 2007. América Latina: la desigualdad y su disfuncionalidad. In: MACHINEA, J. L., SERRA, N. Visiones del desarrollo en América Latina. Santiago do Chile: CEPAL, p. 231-245.

MAINWARING, S., PÉREZ-LIÑAN, A. 2003. Level of development and democracy: Latin American exceptionalism, 1945-1996. Comparative Political Studies, vol. 36, nº 9, p. 1031-1067.

MAINWARING, S., PÉREZ-LIÑAN, A. 2007. Why regions of the world are important: Regional specificities and region-wide diffusion of democracy. In: Regimes and Democracy in Latin America. Theories and Methods. New York: Oxford University Press, 199-229.

MELTZER, A. H., RICHARD, S. F. 1981. A rational theory of the size of government. Journal of political Economy, vol. 89, n 5, p. 914-927.

MONASTERIO, L., EHRL, P. 2015. Colônias de povoamento versus colônias de exploração: de Heeren a Acemoglu. Revista Análise Econômica, vol. 27, nº 72, p. 213-239.

MULLER, E. N. 1988. Democracy, economic development, and income inequality. American Sociological Review, vol. 53, $\mathrm{n}^{\circ}$ 1, p. 50-68.

PERSSON, T., TABELLINI, G. 2003. The economic effects of constitutions: what do the data say? MIT Press.

RAVALliON, M. 2016. The Economics of Poverty: History, Measurement, and Policy. Oxford University Press. https://econpapers.repec.org/RePEc:oxp:obooks:9780190212773

RAVALLION, M., CHEN, S., SANGRAUlA, P. 2008. Dollar a day revisited. The World Bank. ROBINSON, J., ACEMOGLU, R. 2012. Why nations fail. New York: Crown Publishing Group. 
SAINT-PAUL, G., VERDIER, T. 1993. Education, democracy and growth. Journal of development Economics, vol. $42, \mathrm{n}^{\circ}$ 2, p. 399-407.

SAMPAIO Jr, P. A. 1999. Origem e desdobramento da crise da teoria do desenvolvimento na América Latina. São Paulo em perspectiva, vol. 13, n $1-2$.

SANTOS, W. G. dos. 2001. A Democracia e seu Futuro no Brasil. Rio de Janeiro. Manuscrito.

SCHUMPETER, J. A. 1942. Capitalism, socialism and democracy. J. Econ. Literature, 20, 1463.

TAVARES, J., WACZIARG, R. 2001. How democracy affects growth. European economic review, vol. $45, n^{\circ} 8$, p. $1341-1378$.

TIMMONS, J. F. 2010. Does democracy reduce economic inequality? British Journal of Political Science, $741-757$.

TODARO, M. P. 2002. Economic development. Pearson Education India.

VON BRAUN, J., GROTE, U. 2000. Does Decentralization Serve the Poor? Center for Development Research, University of Bonn, Germany.

WILLIAMSON, J. G. 2015. Latin American Inequality: Colonial Origins, Commodity Booms or a Missed Twentieth-Century Leveling? Journal of Human Development and Capabilities, vol. 16, $\mathrm{n}^{\circ}$ 3, p. 324-341. 